\title{
PathoDNA, an Advanced Diagnostic for Lyme Disease \& Co-Infections Utilizing Next Generation DNA Sequencing with Greater Sensitivity and Selectivity than ELISA/Western Blot
}

\author{
Robert Schettig, Ruth Tan-Lim, Daniel Warren, Zachary Poteet, Rory Sears, Michael Hummel, \\ Connor Coffin, Kendra Quart, Christopher Aussems, Ronald Matthias Jr., David Medina, \\ Matthew Klein, Gayle Roberts, John C. Oertle III, Dino Prato \\ Envita, Scottsdale, USA \\ Email: RobertS@envita.com,DinoP@envita.com
}

How to cite this paper: Schettig, R., Tan-Lim, R., Warren, D., Poteet, Z., Sears, R., Hummel, M., Coffin, C., Quart, K., Aussems, C., Matthias Jr., R., Medina, D., Klein, M., Roberts, G., Oertle III, J.C. and Prato, D. (2021) PathoDNA, an Advanced Diagnostic for Lyme Disease \& Co-Infections Utilizing Next Generation DNA Sequencing with Greater Sensitivity and Selectivity than ELISA/Western Blot. Advances in Infectious Diseases, 11, 405-429.

https://doi.org/10.4236/aid.2021.114037

Received: October 26, 2021

Accepted: December 11, 2021

Published: December 14, 2021

Copyright $\odot 2021$ by author(s) and Scientific Research Publishing Inc. This work is licensed under the Creative Commons Attribution International License (CC BY 4.0).

http://creativecommons.org/licenses/by/4.0/

\begin{abstract}
The controversial subject of chronic Lyme disease has occupied medical discourse for years while contributing to unprecedented patient suffering in the United States and abroad. A general misunderstanding of Lyme disease and overconfidence in the Center for Disease Control's (CDC) recommended two-step test for Lyme disease has led to misdiagnosis and incorrect treatment over the years. This leads to increasing medical expenses and worse outcomes for patients. The two-step test, an ELISA immunoblot followed by a confirmatory Western blot, yields accuracy rates as low as $29 \%$ for acute Lyme disease and $75 \%$ for chronic Lyme disease. While these practices have been a staple of microbiology for decades, these accuracy rates are unacceptable for diagnostic tests when better technology is available. PathoDNA, a Next-Generation DNA sequencing test for Lyme disease and other tick-borne pathogens, achieves accuracy rates of $98 \%$ for B. burgdorferi and $95 \%$ or greater for other common tick-borne pathogens with superior sensitivity and selectivity. PathoDNA is a Clinical Laboratory Improvement and Amendments (CLIA)-validated laboratory test that achieves these results utilizing Next Generational DNA Sequencing and a proprietary bioinformatics database. Thus, it allows for rapid results and specific identification of tick-borne illnesses. In this article, we will compare this promising technology against the existing standards for diagnosing and testing Lyme disease. We believe that PathoDNA can set a new standard for identifying Borrelia and diagnosing Lyme disease along with other tick-borne infections.
\end{abstract}




\section{Keywords}

Lyme Disease, Ticks, Tick-Borne, Borrelia, Sequencing, Diagnosis

\section{Introduction}

Every year, thousands of people across the United States go undiagnosed with infectious diseases. This is especially true of those suffering from Lyme disease and associated tick-borne infections. Unidentified infections result in prolonged stays in the hospital, more frequent readmission, and increased mortality and morbidity through delayed or inappropriate treatment [1] [2]. Unidentified infections have also been implicated as a potential cause of some cancers, autoimmune conditions, chronic fatigue syndrome, and many other acute and chronic disorders [3]-[9]. Correctly identifying an infection allows for the use of more closely targeted treatments and narrow-spectrum antibiotics. This is desirable because overuse of broad-spectrum antibiotics leads to the emergences of new, resistant strains of pathogens [10].

One of the most difficult to detect and diagnose infectious diseases that pose a threat to public health is Lyme disease. Lyme disease is the most common vector-borne disease in the United States, with the majority of cases occurring in the Northeast, mid-Atlantic, and upper Midwest regions [11]. Lyme disease is already a public health threat in both Europe and the USA and is currently poised to become the number one spreading, vector-borne epidemic in the world [12] [13]. Tick-borne illnesses increased from 48,600 cases in 2016 to 59,300 in 2017 [14]. A steady increase in the cases of reported Lyme disease has continued to be observed, which appear to be driven by both milder winter temperatures and extended spring and summer days, both of which benefit the primary vector for Lyme, Ixodes scapularis.

Across the United States, tick species capable of carrying Lyme disease are now found in nearly $50 \%$ of all counties [14]. In 2018, the Borrelia burgdorferi carrying Asian long-horned tick was newly identified in several US states [15]. The CDC has measured an estimated incidence rate of about 30,000 reported cases of Lyme disease each year between 2008 and 2015. This rate increases year after year and is triple the rate from 1992. The CDC acknowledges that Lyme disease is often misdiagnosed and is severely underreported in the United States [11]. Federal scientists have suggested that the actual incidence rate of Lyme disease is as much as ten times the number reported between 2008 and 2015 [11]. Thus, the actual incidence rate may exceed 300,000 cases per year [11].

A survey of seven of the largest commercial laboratories in the states (representing $>76 \%$ of all Lyme disease tests performed in the same year) found that approximately 3.4 million Lyme disease tests were conducted on 2.4 million samples in 2008 at a cost to patients and health care providers of $\$ 492$ million [16]; Two-tiered assays as mandated by the CDC accounted for $62 \%$ of all tests 
performed. This is actually higher when including tests performed individually (such as an initial ELISA upon arriving at a hospital), which were not included in this figure. The same researchers estimated that these reported tests corresponded to about 240,000 to 444,000 infected people for 2008 ; this aligns roughly with the estimate released by the CDC if only $10 \%$ of cases were reported to the official count.

In New York, tick expansion coincided both spatially and temporally with warmer temperatures; the researchers there found that mild winter days were strongly predictive with summer encounters with I. scapularis [17]. Europe has reported a steady increase in Lyme infections for the past 20 years, with more than 360,000 patient cases recorded [18]. There is evidence suggesting that the infection rate is even higher in Europe than the reported number. Sykes et al. found a much higher infection burden in Western Europe alone than the World Health Organization (WHO) figure would indicate 232,000 cases per year on average [19]. This result gives additional credence to the idea that the real infection rate in the US is much higher than the reported numbers [11]. A 2013 conference on Lyme disease released numbers from a self-reported survey which indicated a $0.3 \%$ infection rate across the US for the year 2012, which would amount to well over 900,000 people if it were applied to the entire country [20]. While the statistics from a self-reported survey should be taken with a grain of salt, it is an indicator that more honest and professional surveying of the actual disease rate is needed, given the wide discrepancy between reported infection rates for Lyme disease.

The increasing rates of Lyme disease seen in Figure 1 degrade public welfare and put individual lives in danger. It also places a burden on the health system, which is underreported because of commonly undiagnosed or misdiagnosed Lyme disease cases. An observational cohort conducted between 2000 and 2013 showed that incorrect diagnoses and unnecessary treatments were common for Lyme disease patients [21]. If the patient presents without a rash, the patient stands a 54\% chance of being misdiagnosed [22]. When patients receive treatments that provide no benefit to them that places a burden on the healthcare system and contributes to inefficiencies of the system as a whole.

A 2015 study attempted to measure long-term issues Lyme patients faced and the increased cost of care associated with Lyme disease. Adrion et al. noted that despite standard treatment protocols that insist that Lyme disease treatment ends after about eight weeks, patients were returning to the doctor with persistent symptoms. This would then be followed by multiple additional rounds of testing and re-treatment. On average it is estimated that "people with Lyme disease cost the system $\$ 2968$ more than matched controls, and they cost the health care system about $\$ 1$ billion a year" [23]. Other estimates have been higher. With corrections for inflation, a 2006 study found that the mean annual cost of late Lyme disease per patient was $\$ 20,502$ [24] [25].

Lyme disease also comes with a significant societal cost. Accounting for lost productivity and the cost of the health care itself, a study from the Netherlands 


\section{Confirmed and Probable Lyme Disease Cases}

by state surveillance category and year - USA, 1992-2015

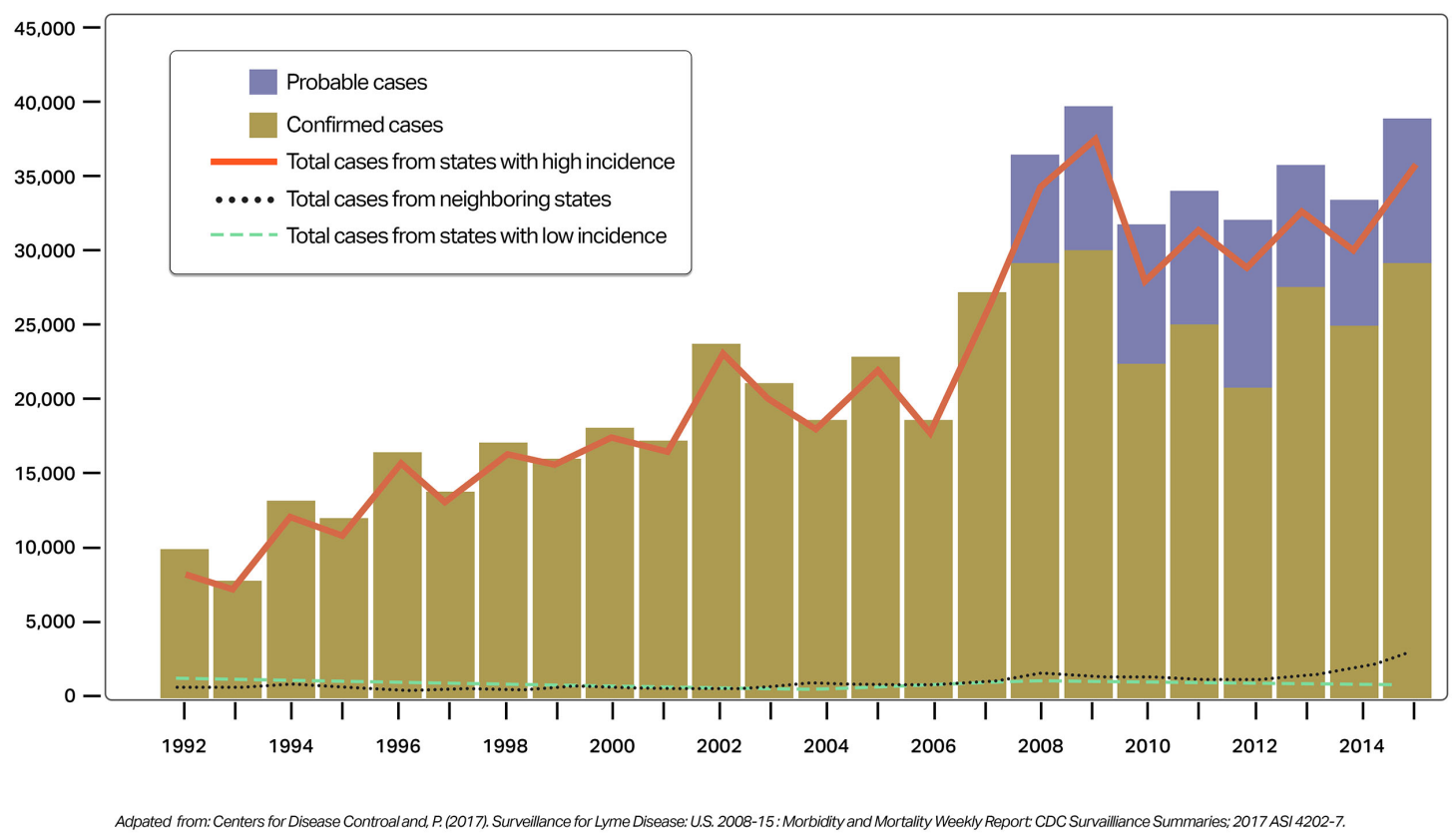

Figure 1. The rate of confirmed and probable cases of Lyme disease in the United States as reported by the CDC between 1992 and 2015.

estimated a total cost of $€ 19.3$ million to the nation in 2010 resulting from Lyme disease. Forty-eight percent of this loss originated from the cost of health care and lost production from sick workers, with the cost to the patient amounting to $4 \%$ of the total economic impact. The authors recommended increased funding for preventative measures for Lyme disease due to the societal impact of the disease [26]. In another Netherlands study in 2010, Lyme disease resulted in 10.55 Disability Adjusted Life-Years (DALY) per 100,000 people. DALY's are a measure of the years lost in a person's life from disease burden that results in ill-health, disability, and early death [27]. Most of these DALY resulted from persistent Lyme disease symptoms that degraded quality of life. The increasing epidemic of Lyme and other tick-borne illnesses demands utilizing improved detection technology and diagnostic resources.

However, Borrelia burgdorferi has been historically difficult to detect. This is because the organism contains many defensive countermeasures including interference with the active immune system. For example, Borrelia evades lysis through the complement immune response by expressing a factor H-binding protein identified by Kraiczy et al. [28]. Xu et al. write about the ability of Borrelia to change the expression of its outer surface proteins in order to evade the immune system during initial infection and then later during humoral response [29]. Lyme disease may also be confused for other conditions while making a diagnosis [30]. There have been cases where Lyme has been misdiagnosed as 
Lymphoma or dermatomyositis among other conditions [31] [32]. Regularly, patients who present with erythema migrans(the bull's eye rash commonly associated with a Lyme-infected tick bite)are automatically considered infected and prescribed a simple antibiotic treatment without confirmatory testing [33]. Meanwhile, patients who do not present with the rash, but may have a history of tick exposure or persistent symptoms usually undergo CDC-mandated ELISA and Western blot testing [34]. These tests do not look for the organism B. burgdorferi directly, however, but rather indirectly through the human antibody response to the pathogen [35] [36] [37].

The search for Lyme disease through indirect antibody tests may explain some of the inaccuracies we observe with ELISA/Western blot tests. Another contributing factor may arise through multiple coinfections that tend to occur alongside a primary Lyme disease infection [38]. Another reason for the ongoing lack of accuracy in Lyme testing may be lack of funding for Lyme disease research. NIH spent only $\$ 63$ per patient in 2018 towards research [39]. In comparison to a different vector-borne disease, NIH spent $\$ 118,823$ per patient for Malaria and $\$ 13,600$ per patient for West Nile virus in 2018 [39].

In this article, the multiple problems with current standard testing regimen for Lyme disease will be examined in greater detail by the technology used to scan for the pathogen [40]. The primary concern over the standard diagnostic algorithm is that it relies on expression of relevant antibodies in the patient. This is unreliable in the case of early Lyme disease since it takes several weeks for an antibody response to develop, producing a high rate of false negatives [40] [41]. Additionally, in late Lyme disease, the immune system can become compromised which also limits the antibody reaction; in the late stage, two-tiered testing with ELISA and Western blot may miss as much as $44 \%$ of infected patients [42]. In addition to becoming immune-compromised, Borrelia antibodies frequently become bound up in circulating immune complexes leading to the inaccuracies measured in standard immunoassays [43]. Also, tests prepared in the US typically only attempt to detect a few prevalent species and are usually incapable of determining foreign infections from strains such as Borrelia miyamotoi [44] [45]. In measuring the effectiveness of ELISA test kits meant for the diagnosis of Lyme disease, Ang et al. found that the sensitivity of the tests varied wildly from $20.9 \%$ to $97.7 \%$ [46]. Conventional methods of direct detection of the bacterium such as PCR have proven difficult in the past because Borrelia typically manifests with low bacterial counts that are dispersed quickly throughout the entire body [47]. The lack of an effective diagnostic method, especially in the case of early Lyme disease, can mean missing a vital treatment window in which the infection might be cured before it becomes chronic.

The challenges Lyme disease and other difficult-to-detect infections pose to modern health require a modern technological solution. The next step forward appears to be Next-Generation Sequencing (NGS) which offers big advantages in sensitivity over antibody tests like ELISA and Western blot and even over newer 
PCR-based detection protocols. In this report, we review the current use of detection methods including ELISA and Western blot, and how they compare to the new technology of NGS.

\section{Western Blot}

Western blot has been a staple technique in biochemistry since the 1980s [48]. It is a firmly established tool to detect and analyze proteins. And while as a forensic and diagnostic tool, Western blot is still considered a pillar of diagnostic science, it is also a 40-year-old technology. As newer and more advanced processes come along, there has always been resistance to the displacement of accepted methods. In the face of that resistance, it can be useful to break down exactly what these older processes like Western blot and ELISA are, how they work, and their limitations.

Western blot primarily detects single proteins qualitatively, especially from complex mixtures of multiple proteins. The test is only semi-quantitative since the size and color of a protein band correlate roughly with the amount of protein present. While strong and faint bands are easy to distinguish, it is important to remember that smaller differences can be more difficult for an examiner to differentiate and introduce subjective interpretation into results. Western blot is more helpful for determining the presence or absence of a particular protein than obtaining more detailed information in practice. Liang et al. write that subjective interpretation of the banding patterns produced by Western blot can potentially introduce errors in reporting results [49].

This qualitative testing ability suits the needs of many doctors in making certain kinds of diagnoses. However, Western blot meets challenges in circumstances where there are weak or muddled protein signals which can make or break an early diagnosis. In the case of Lyme disease, Western blot is less than ideal for making an early diagnosis because the IgM response, the key factor for which Western blot is looking for, is insensitive, nonspecific, or both in the first weeks following infection [50]. It is not until late Lyme disease that Western blot can make a concrete diagnosis.

In the case of Human Immunodeficiency Virus (HIV), Western blot is being phased out for use in detection and diagnosis entirely. Once the gold standard, Western blot is being gradually removed from the recommended HIV diagnostic algorithm due to its inability to detect early HIV infection, the length of time the testing requires, and a tendency to misclassify HIV-2 infections as HIV-1 [51]. Interestingly, diseases such as Lyme, syphilis and lupus can cause false positives in the ELISA test for HIV infection [52]. The similarity here between HIV and Lyme is striking because advanced Lyme disease can also result in an immune-compromised state [53].

In North America, the species, Borrelia burgdorferi is associated with Lyme disease [54], and Western blot tests produced in the US reflect that. Outside North America, however, the situation can be very different. For example, in 
Europe, there is a much more significant species heterogeneity among Borrelia with at least four different species present on the continent [55]. When testing a group of 64 European Lyme disease patients, the accepted 2-step US assay (ELISA followed by Western blot) was only 52\% sensitive compared to $81 \%$ for the European assays $(P=0.0007)$ [56]. The most remarkable difference between the two tests was observed for an early-stage disease where sensitivity for the US test fell to $20 \%$ with the European test at $55 \%$ sensitivity $(P=0.05)$ [56]. Lyme disease is known for being difficult to detect, especially in the early stages, and it is worth noting that even the European test had relatively low sensitivity in the early stage, when it is most important to make a diagnosis. The necessary Borrelial surface antigenic diversity required for a more accurate test is a challenge for serodiagnostic tools like Western blot, which can have excellent specificity but may require additional arrays to scan for multiple similar serotypes [57] [58].

Some attempts have been made to improve the sensitivity of Lyme disease tests utilizing Western blot. Igenex, an immunology lab based in California, produces a Lyme Western blot test which they claim has greater sensitivity than the CDC-recommended test. This is primarily accomplished by recognizing additional protein bands as confirmation for the presence of Borrelia [59]. This expanded definition of a confirmatory Lyme disease test has led some to criticize the test for increasing the risk of false positives [60]. Igenex explains that one reason why their specified band patterns differ from the CDC definition of Lyme disease is that the extra bands may represent common coinfections that coincide with Borrelia infections. Lyme disease is difficult to detect on its own, so looking for more detectable coinfections that are associated with Lyme disease could be a valid strategy for making the diagnosis.

Still, clinicians should strive for a test with better accuracy. Even expanding the definition of a positive Lyme disease result as Igenex has remains controversial among conventional and integrative doctors. Aguero-Rosenfeld et al. suggested in their 2005 review of techniques used to diagnose Lyme disease that the sensitivity of the two-tiered system is likely even lower, presenting a range of $29 \%$ - 40\% sensitivity for early Lyme disease [61].

\section{Elisa}

Enzyme-linked immunosorbent assay, or ELISA, has been around since first described by Engvall and Perlmann in 1972 [62]. Like Western blot, it endures as a key biochemical tool for the qualification and quantification of specific proteins. Clinicians continue to rely on ELISA to make many diagnoses, especially of infectious diseases. However, being a nearly 50-year-old technology, ELISA shares several of the same faults of Western blot. It remains a good tool for protein identification, but newer and better options are becoming increasingly available where diagnostics are concerned.

ELISA involves the immobilization of an antigen onto the bottom of several 
testing chambers, typically a titered assay, and the use of antibodies generated to specifically adhere to the chosen antigen. Enzymes are attached to the antibody so that when the substrate for the enzyme is fed into the test chamber, a visible reaction such as a change in color is produced. There are several variations of ELISA, including direct, sandwich, and reverse ELISA, but they all rely on this underlying process. On paper, this seems like a fool-proof detection process, but there are several hidden weaknesses when trying to use ELISA as a diagnostic test.

When there are problems with ELISA, many of them come down to the immobilization technique. For instance, in direct ELISA, when extracted serum is dried to the bottom or sides of a testing well, there will usually be many other proteins aside from the one of interest in the mix and in unknown quantities. There may be only a tiny portion of protein exposed on the surface where antibodies can react. This can cause weak enzyme signals and equivocal results. Sandwich ELISA is an attempt to solve this by immobilizing capture antibodies instead so that they can pull target antigens out of the solution, but this technique has its issues, often needing separate validation due to the risk of false positives [63].

In the case of Lyme disease, the sensitivity of ELISA can vary substantially, lending credence to the reputation of this disease being elusive and difficult to diagnose. A newer version of ELISA, called C6 ELISA, has been proposed to replace the two-tier test with ELISA and Western blot. When attempting to detect Lyme in early-stage disease, patients exhibiting erythema migrans, C6 ELISA featured a sensitivity of $66 \%$ with conventional two-tier verification exhibiting a sensitivity of only $35 \%$ [64]. This shows that there is room for significant improvements in the basic technology, especially considering that in this case, the researchers achieved greater sensitivity for early Lyme disease with a single test than a two-tier process with both ELISA and Western blot.

Assays for detecting Lyme with ELISA are consistently low. EUCALB (European Concerted Action on Lyme Borreliosis) states that the specificity of assays should be over $90 \%$ for a screening test and over $95 \%$ for a confirmatory technique. A review of several commercially available assays for Lyme found that many of the tests had sensitivities all over the place, with an overall specificity of $67 \%$. The same researchers also found that patients with malaria often elicited false positives in these standard assays [65]. With the performance of ELISA and Western blot both lackluster in detecting Lyme disease, it is about time patients and physicians have improved technology.

\section{PCR Standalone Tests Are Problematic}

PCR (Polymerase Chain Reaction) has been offered as a potential standalone test for Lyme borreliosis, however, its performance leaves much to be desired in a diagnostic test. PCR is a very useful technology used for the amplification of DNA in a sample or creating copies of DNA segments from a small amount of 
starting material. The replication process is exponentially based upon the number of heating and cooling cycles that are run, and a single DNA sequence can be multiplied into millions or billions of copies [66]. This is frequently necessary for biotechnology to increase the concentration of DNA in a sample to levels that are detectable and usable by humans.

PCR does not replicate all of the DNA found in a particular sample. Rather, the process is controlled by primers, small segments of DNA which bound the forward and reverse ends of the segment of DNA that is desired to be replicated. Only the region of DNA between the primers is replicated, leaving all other DNA in the sample at a very low concentration. PCR is considered to be extremely sensitive to contamination by foreign DNA leading to spurious DNA products considered to be a chief limitation of the technology [67]. It seems natural to assume that an extremely sensitive technique in biotechnology would lead directly into a very sensitive diagnostic test, but making that transition is more complicated than it would appear at first glance.

Skogman et al. evaluated two different PCR diagnostic tests for Lyme disease $\left(\right.$ Taqman $^{\circledR}$ and LUX ${ }^{\mathrm{TM}}$ ) among Swedish children afflicted with Lyme borreliosis [68]. The tests were retrospective, allowing the researchers to categorize the children into groups of "Definite," "Possible," and "non-Lyme" in terms of their Lyme disease status; another group of children not exposed to Lyme disease was used as a control group. The PCR tests identified only a small portion of the definite Lyme cases as positive for Lyme disease (5\%) while changing one child from the "Possible" group to "Definite" and another child from the "non-Lyme" group to "Definite" [68]. The conclusion that Skogman and her colleagues drew from the results was that these two PCR tests were better suited to a complementary role in Lyme disease testing due to its very low sensitivity but could still remain useful for its ability to catch certain cases that would otherwise not be classified as a Lyme infection.

One explanation for this stunning lack of sensitivity for PCR tests designed specifically for Lyme disease is that PCR is reliant on the choice of primers/probes for the test. An incorrect or suboptimally designed set of primers would result in failed PCR reactions. Another possible explanation is the suspected low population of spirochetes in cerebral spinal fluid which Skogman was using for her study [68]. Other studies offer a range of sensitivities for Lyme PCR detection depending on the origin of the source tissue. Dessau et al. report a median of $69 \%$ sensitivity with the best results for biopsies of erythema migrans and the worst for cerebrospinal fluid with sensitivity approaching $40 \%$ [69]. Under certain circumstances, sensitivity is reported to be higher [70] [71], however, the wide range of reported sensitivity for this test is a major concern when trying to make a firm diagnosis. PCR is undoubtedly an invaluable technology for a large number of procedures dealing with DNA, but as a diagnostic test, there are certainly better options such as Next-Generation DNA Sequencing. 


\section{PathoDNA: The Next Step in Lyme Disease Detection/Diagnosis}

Next-generation DNA sequencing (NGS) offers numerous advantages over the older technologies of ELISA and Western blot. PCR and ELISA can only test for a single organism at a time, requiring the physician to make a hypothesis, or best guess, at what the pathogen might be first [38]. Due to many possible pathogenic organisms, this narrow identification can lead to negative results and the patient enduring ongoing tests searching for the correct diagnosis. A diagnostic test that could scan for pathogens with a broad scope and result in an accurate identification would revolutionize microbial diagnostic testing. Next-Generation DNA Sequencing (NGS) utilizing bioinformatics is the right technology needed for many clinicians searching for more accurate and efficient ways to identify the etiology of their difficult-to-diagnose patients with unknown or complex infectious diseases.

Hypothesis-free testing can drastically speed up the diagnosis process for physicians since there would not be a need to test for each potential infection individually or wait for results before submitting subsequent tests. Broad identification is made possible by sequencing all of the DNA and RNA present in a sample through an NGS technique called "Shotgun Sequencing" [72]. A more targeted approach is also possible. For example, the clinician can use primers from conserved 16S ribosomal RNA or internal transcribed spacer sequences for either universal bacterial or fungal detection, respectively [73] [74]. This sort of narrowed focus allows for species identification of these microorganisms. Analyzing the genetic code of identified pathogens allows for identification of the specific strain [75] [76] and even prediction of possible drug resistance [77], both critical pieces of information for the diagnostician.

Another important quality of NGS is the ability to quantify the presence of an infection. ELISA and Western blot are only capable of positive or negative results, but NGS can measure the amount of bacterial DNA in a sample so that the infection can be tracked over time by a physician. This is done through counting of sequenced reads [74]. Tracking the progress or regression of the infection over time allows the attending physician to determine if the infection is responding to treatment or not without having to rely only on signs and symptoms. This is especially useful in patients with multiple infections where it can be challenging to determine which pathogen is causing which symptoms. Quantifying infections in this way can make all the difference for patients by objectively tracking the amount of infectious material in a sample and avoiding confounding circumstances such as when one infection is advancing while another is responding to treatment.

PathoDNA is a lab-developed test specifically designed to detect and diagnose Lyme disease and associated coinfections. It utilizes an NGS technology known as "shotgun sequencing" and a proprietary database of known bacterial DNA profiles. Shotgun sequencing is the process of sequencing all DNA found in a 
sample rather than using a marker like $16 \mathrm{~S}$ to compare specific regions of DNA. There are two major approaches to shotgun sequencing, metagenomics, which examines present DNA from a sample, and metatranscriptomics, which sequences the mRNAs present in a sample. PathoDNA takes a metagenomic approach to classifying DNA.

Figure 2 illustrates the 4 main steps in the PathoDNA diagnostic process. The test was designed to process either a blood or a urine sample from a patient. The inclusion of the ability to test urine for spirochetes and coinfections is important since there is data suggesting that these organisms reside in greater concentrations inside the urine and bladder tissue of the host [78]. DNA is extracted and purified from the patient sample and then sent through a DNA amplification process. AmpliSeq primers are used following the AmpliSeq Library protocol according to CLN-00001-SOP [79]. During this step, the samples are normalized using the AmpliSeq Equalizer Kit before being pooled for emulsion PCR. The PCR product is loaded onto enriched beads before being placed onto a chip for DNA sequencing. Sequencing is performed on the Ion Torrent S5 sequencer [79]. Specialized software is used to trim and filter sequences as they are generated because the shotgun sequencing process produces many small sequences of DNA that need to be identified and sorted into a library for analysis. It is important to remove reads from the sequence output that map to the known human genome, leaving only non-human DNA for data processing. DNA sequencing by the shotgun process requires that reads be pooled according to probable species and counted. Software counts the number of reads generated by the sequencing process and uses this information to assign probability thresholds for matching sets of amplicons. The number of reads of a particular sequence that is generated by the sequencing process corresponds to the quantity of DNA that was present in the original sample [79].

Metagenomics is often used to understand the functional component of a community of organisms (i.e., an infection). This can be inferred by comparison

\section{DNA Extraction from Blood or Urine}
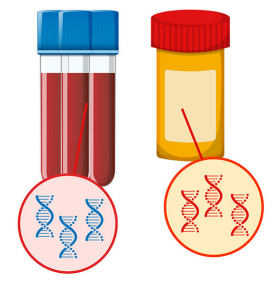

DNA is extracted and purified from either blood or urine. Intracellular bacteria can be easier to detect from urine than from blood.

\section{DNA Amplification} for Tick-Borne Infections

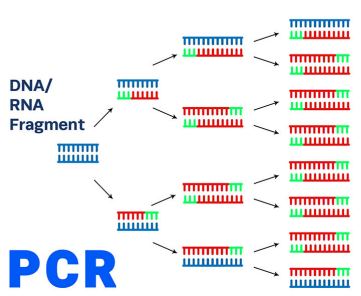

DNA fragments are amplified using PCR so that the DNA signal is strong enough to be detected during the sequencing process.

\section{Shotgun Sequencing}

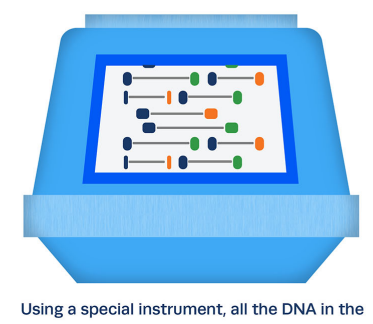

immobilized fragment library is sequenced at the same time. Raw DNA sequence data is compared against a custom DNA library for Lym disease and associated coinfections.

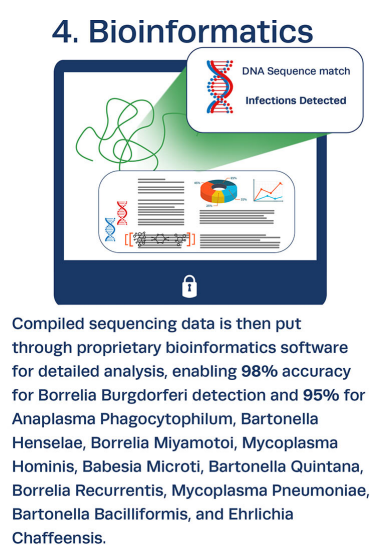

Figure 2. The four main steps of PathoDNA, starting with blood or a urine sample. PCR composes the second step where the DNA is amplified. Next-generation DNA sequencing is the third step where all of the DNA that was amplified in step 2 is sequenced. Step 4 compares the results of the DNA sequencing against a proprietary database containing known sequences for Borrelia and other tick-related infectious organisms. 
of the results against database records such as KEGG (Kyoto Encyclopedia of Genes and Genomes), a pathway database, or COG (Clusters of Orthologous Groups of Proteins), a database containing functional categories [80] [81]. The information gleaned in this way should be taken with a grain of salt, however. Metagenomics, by its nature, involves comparing different parts of different genomes against one another. This makes adequate classification a crucial step in obtaining legible data. A good reference library is essential in metagenomics, and a limited reference sequence library can produce unreliable results. This is a problem PathoDNA seeks to resolve with its customized reference library specifically designed for the different strains of Lyme disease and associated coinfections such as Bartonella and Mycoplasma.

Genetic First developed PathoDNA in association with Envita Medical Centers, a Center of Excellence for the treatment of chronic Lyme disease, utilizing clinical pathology laboratories, bioinformatic experts, and specialized Lyme disease physicians. Data from over 1000 Lyme disease patients were compiled to form the DNA sequence library that PathoDNA relies upon. Genetic First succeeded in bringing PathoDNA through the CLIA validation process to ensure that the test is sound and backed scientifically with strong evidence for its veracity and repeatability. PathoDNA was designed to be ordered through physicians so that a doctor can review results with the patient and provide assistance and understanding rather than allow the patient to order and review test results on their own which can lead to poor follow-up support. It is hoped that PathoDNA will meet the demand for new and high-tech solutions for difficult diseases like Lyme and other tickborne infections. Below, Figure 3 illustrates a list of organisms detected by PathoDNA that cause Lyme disease or its associated coinfections.

\section{Organisms Detected by PathoDNA}

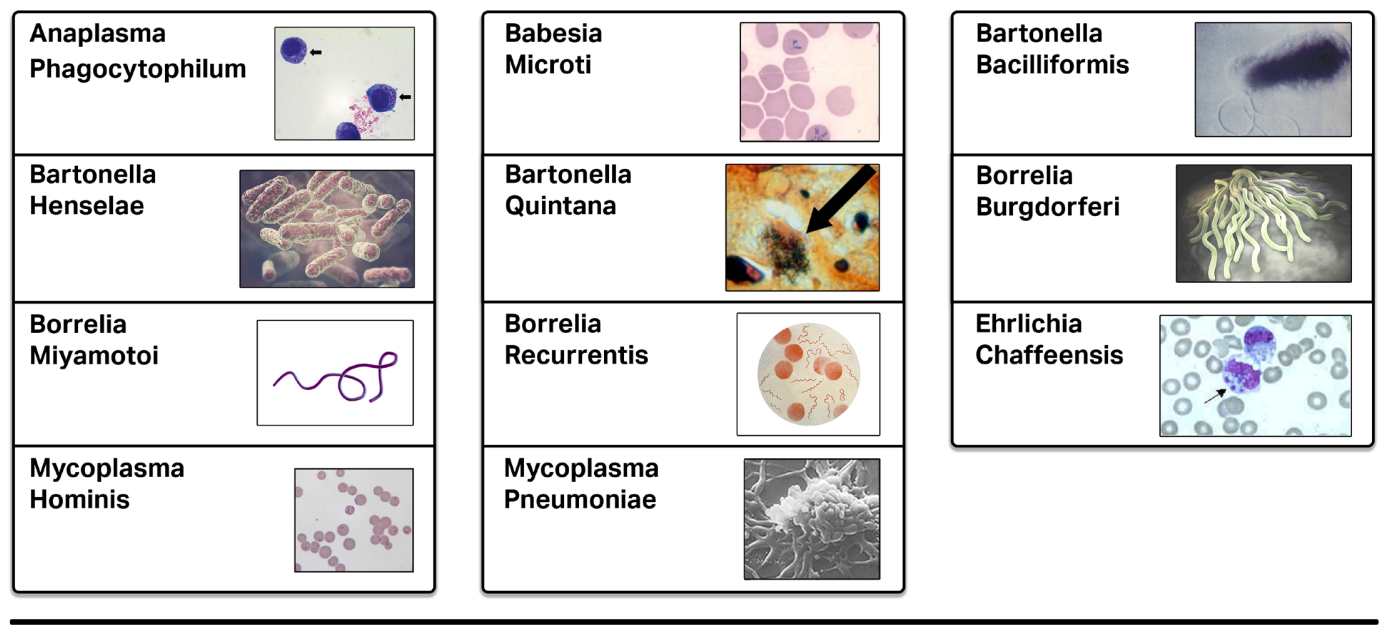

PathoDNA is a lab-developed test designed specifically to detect and diagnose Lyme disease and associated confections. It utilized a NGS technology known as "shotgun sequencing" along with a proprietary database of known bacterial DNA profiles.

Figure 3. A list of organisms detected by PathoDNA [79]. 
This article has discussed some of the shortcomings of the existing test protocol for Lyme disease dictated by the CDC involving the older technologies of Western blot and ELISA. DNA sequencing is still emerging onto the market for patients to gain relatively easy and affordable access to more sensitive and accurate diagnostics. Understanding the legal status of some of these tests can be tricky at times. The FDA classifies in vitro diagnostic tests developed and performed by a single laboratory to be a "Laboratory Developed Test" (LDT). These are also sometimes referred to as "in-house" or "homebrew" tests. LDT's can be FDA-approved or CLIA validated, though due to poor enforcement, sometimes LDT's are neither of these.

So-called "alternative tests" have been available to patients willing to go outside the medical insurance system for many years, but it is frequently difficult for the average person to distinguish between science-backed medicine and pseudo-scientific or non-scientific tests and treatments. Fortunately, the Clinical Laboratory Improvement Amendments (CLIA) program offers a way for consumers to know that the tests laboratories offer are accurate and reliable.

LDT's and other in-house developed tests which have not received FDA clearance or approval are prohibited by CLIA from releasing any test results until the laboratory establishes certain validity and performance characteristics described by 42 CFR 493.1253(b)(2). This is also known as "CLIA Validation." While the Code of Federal Regulations requires CLIA validation for non-FDAapproved laboratory tests, the law is not frequently enforced, leading to a slew of dubious diagnostic tests being present across the country. Because of this largely unregulated environment, both doctors and patients need to be aware of the importance of test validity in ensuring that they use sound diagnostics to make critical medical decisions.

LDTs have an important and recognized place in the US health care system. In the FDA's own words, "LDT's are important to the continued development of personalized medicine." The FDA was granted authority to monitor and regulate laboratory tests in 1976, but LDTs have grown far more advanced in the decades since then. While there are FDA-approved tests, the government recognizes the innovative role that independent hospitals and laboratories play in providing better health care. That is not to say that there is no oversight of LDT's at all. Even when an independently developed test is not FDA-approved, the laboratory must still demonstrate the competency of both the staff and equipment present in the lab, evidence supporting the efficacy of the test, and that results are not falsified in any way. With these safeguards in place, the FDA has made the wise decision to allow LDT's in the marketplace to fill unique health care niches better and prevent delays in medical treatment. More patients would be better informed to learn that even when a lab test is not FDA-approved, it is still subject to oversight by the FDA to ensure its safety and validity. In fact, the FDA is still updating its draft guidances on LDT's which were last done in 2017 [82].

The FDA explicitly features a class system for laboratory tests and medical de- 
vices ranging from potentially dangerous and highly regulated tests to minimally dangerous tests which general controls can monitor. The process for obtaining FDA approval, however, is long and onerous for most companies, often requiring an enormous financial cost and taking years to complete. This prevents many ideas for tests, especially ones that are not optimally marketable, from making it to the clinical trial stage [83]. Typically, only commercially manufactured tests, sold as kits to other laboratories, are FDA-approved. This is why the FDA allows LDT's to exist, because there are many niches, especially for uncommon diseases, where a laboratory test might be desirable, yet it would not make commercial sense to seek an FDA approval. In this case, LDT's can still seek a CLIA validation to assure patients and the doctors who use them that the test has been reviewed for veracity and clinical soundness. These distinguishing qualities benefit a patient seeking better health care and avoiding fake products and quackery that are presented as illegitimate substitutes for scientifically backed tests.

By achieving CLIA validation, the PathoDNA diagnostic test demonstrated its clinical validity, a scientific measure of its reliability, reproducibility and validity. The final report submitted for validation reported $98.8 \%$ for the detection of $B$. burgdorferi in blood and urine samples and 95\% accuracy for the detection of other tick-borne pathogens [79]. Test validation was measured with several variables:

- Accuracy of Detecting Single Spike-in of Pathogen DNA in Human Urine.

- Accuracy of Detecting Single Spike-in of Pathogen DNA in Human Blood.

- Accuracy of Detecting Mixtures of Spiked-in Pathogen DNA in Human Blood and Urine.

- Repeatability of Detecting Spiked-in Pathogen DNA in Two Separate Assays from the Same Run.

- Reproducibility of Detecting Spiked-in Pathogen DNA in Separate Assays from Different Runs.

- Selectivity of Pathogen DNA Detection in the Presence of Background DNA not included in the Original Assay.

In each of these categories, PathoDNA achieved passing acceptance criteria for CLIA validation. In fact, $98.8 \%$ accuracy for B. burgdorferi exceeds the threshold by a large margin. This accuracy level is needed and should be expected for a medical diagnostic test rather than the low accuracies of the ELISA and Western blot tests quoted earlier in this article.

This accuracy is gained in large part by applying a specialized reference library for the pathogens that cause Lyme disease along with a list of organisms commonly associated with tick-borne disease. The reference library is a database of known DNA sequences used during the shotgun DNA sequencing process after raw sequencing collection and data processing.

Lastly, PathoDNA was designed to accept either a blood or urine sample from a patient, with separate protocols for extracting DNA from either blood or urine [79]. This is important because pathogens can hide in different excreta from the 
body. An organism present in the blood might not necessarily be detectable in the urine and vice versa. In "Modern Methods of Pathogen Detection" the authors write that direct analysis is often made difficult by low pathogen counts and high organic and inorganic contents in the sample (such as heme in blood) [78]. The author also mentions that NGS represents the next step towards using genome sequencing as a diagnostic tool due to the impressive speed and quantity of sequence data produced by methods such as IonTorrent (which PathoDNA relies upon), although NGS systems are still not being used for routine diagnostics. In the case of urine, small molecules can become enriched to higher content than found in the plasma due to the filtration process of the blood by the kidneys. In this way, urine can represent an ultrafiltrate of the plasma [78]. Some species of intracellular bacteria can be determined more easily from the urine than from blood, such as Mycobacterium. Figure 4 shows that it was far easier for researchers to isolate spirochetes from the bladder tissue than from blood samples. In this way, a comprehensive detection system should examine multiple kinds of sample specimens for a more comprehensive analysis of the patient and a better chance of making an accurate diagnosis.

\section{The Migration Pattern of Borrelia Into Tissue Types}

After mice were infected by intraperitoneal or subcutaneous inoculation or by tick bite, Spirochetes were most frequently isolated from the bladder ( $94 \%)$, followed by the kidney $(75 \%)$, spleen (61\%), and blood (13\%).

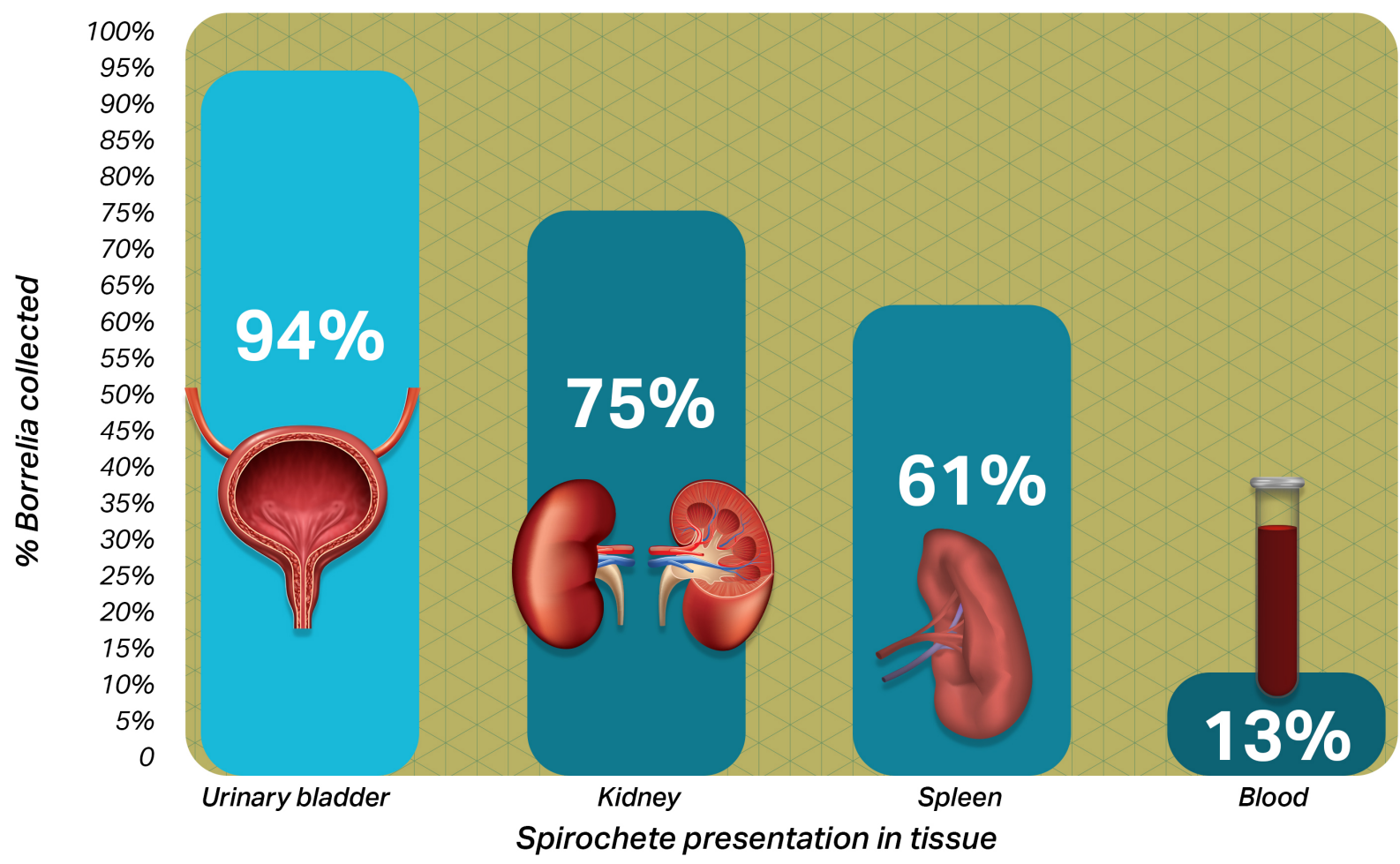

Adapted from: Schwan TG, Burgdorfer W, Schrumpf ME, Karstens RH. The urinary bladder, a consistent source of Borrelia burgdorferi in experimentally infected white-footed mice (Peromyscus leucopus). J Clin Microbiol. 1988;26(5):893-895. doi:10.1128/jcm.26.5.893-895.1988

Figure 4. A graph indicating the relative distribution of spirochetes in the body by tissue type. Researchers were far more likely to isolate spirochetes from the bladder than the blood in murine model. These results strongly indicate that a urine test will be more likely to uncover spirochetes in the body than a blood test. 


\section{Discussion}

In this article, we have taken a closer look at the state of technology used to detect and diagnose Lyme disease and other tick-borne ailments. PathoDNA was developed as a diagnostic test for doctors to address the tremendous need for sound and accurate diagnostic testing in light of the spreading areas where Lyme disease impacts the country. This test uses newer and proven Next-Generation DNA Sequencing technology which offers much greater accuracy for detecting B. burgdorferi and other parasites while maintaining high selectivity. This is a step forward past the CDC-recommended two-tier process for diagnosing Lyme disease utilizing ELISA and Western blot.

We briefly covered how ELISA and Western blot technologies are currently used for the diagnosis of Lyme disease. Both technologies were defined between forty and fifty years ago. The technologies are relatively low cost due to their ubiquitous adoption by the scientific and medical community and existing infrastructure; however, better conclusions can be drawn from newer technology, which is far more sensitive, a feature needed with low-population infections such as Lyme. Even with the combination of both technologies in a two-tier format, ELISA-Western-blot achieves only a 52\% sensitivity for early Lyme disease, well below the FDA's standard for diagnostic tests of at least 95\% [56]. This level of sensitivity means that half of the tests performed come up as a false negative. While two-tier testing eventually increases sensitivity the longer the patient has already been infected, catching Lyme early on and beginning appropriate treatment is critical to affecting a permanent recovery and preventing the development of chronic Lyme disease syndrome. The worst-case scenario here is that a person receives the FDA-mandated version of the test, falsely believes it to be negative and does nothing for the latent Lyme infection, which can cause years of debilitating symptoms ranging from widespread aches and pains and chronic fatigue to neurological deterioration. Unfortunately, this is exactly what is happening and in significant numbers with as many as 300,000 new cases of Lyme each year and all signs indicating that this number will rise in the future [11]. The following Figure 5 illustrates the major difference in accuracy between the two-step, CDC-recommended test, and newer technology like PathoDNA.

Unfortunately, there is a divergence of opinion on how to best address the problem of Lyme disease among medical professionals. This can be seen with Igenex, which is continued to be lauded as a leader of Lyme disease detection. In truth, they only use a more liberal definition of the same test prescribed by the CDC to return a positive result for Lyme [59]. Their tests suffer from the same inherent flaws of technology as the conventional test and are more likely to return a false positive [60]. That is not to say that the CDC is entirely blameless either. For decades, the organization has sat on its hands, recommending and requiring doctors to use a procedure with unacceptably low sensitivity rates by its own standards for other diseases. This may have been fine in the early to mid-90s when no other options were realistically available [84], but miniaturization and automation have now put DNA sequencing technology within the grasp 


\section{Two-Tier ELISA/Western Blot Accuracy vs PathoDNA Accuracy}

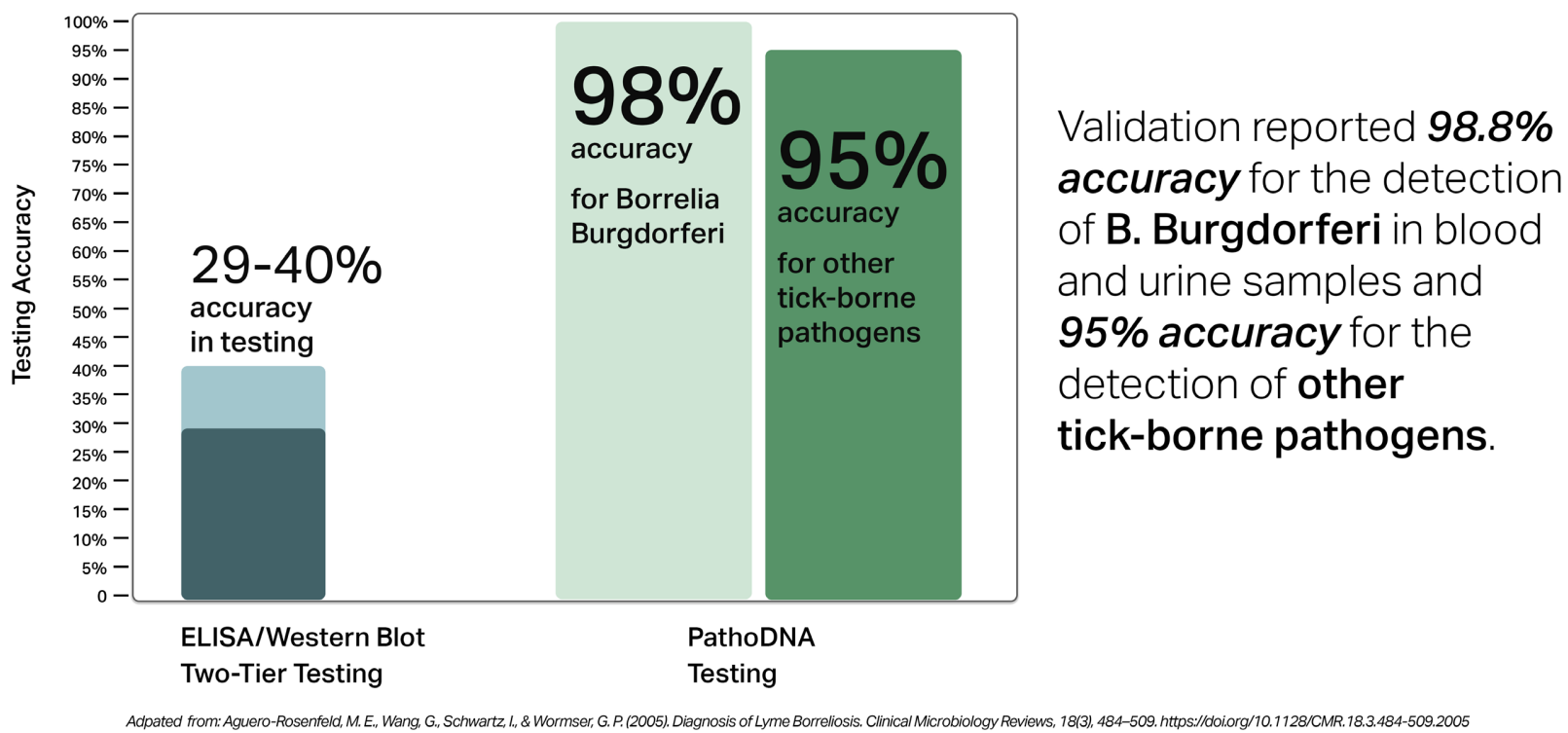

Figure 5. A comparison of the accuracy of the CDC-mandated two-step test for Lyme disease utilizing ELISA and Western blot and PathoDNA with Next-Generation DNA sequencing [61] [79].

of nearly everyone in the country. Issuing guidance for doctors for an inadequate test actively harms tens of thousands of people each year. Health insurance companies base what they will cover on issuances made by the CDC. As long as two-tier testing for Lyme disease is standard in the US, other, better forms of diagnostics will not be covered, which effectively prevents access to adequate care for millions of people in this country. Unfortunately, the CDC has a history of silence concerning past mistakes, and remedies may come years or decades after the fact if they come at all [85].

It is fortunate then that visionary groups like Envita Medical Centers are willing to explore a niche for advanced genetic testing where there is a real health need that is not being met. The leaders at Envita felt it was necessary to step forward to meet the popular demand from both practitioners and patients to fulfill the need for better diagnostic tests. New, better technology should be embraced readily and rapidly for areas where they offer tangible improvements, and existing techniques are either subpar or problematic. Envita's vision is to provide a diagnostic service designed to help patients and doctors, improving health outcomes while lowering medical costs. Better diagnostic technology is key to improving both the patient's and the doctor's experience when dealing with a potential case of Lyme disease as well as associated coinfections.

The truth is that Next-Generation Sequencing has already demonstrated both efficiency and reliability for the detection of infectious organisms in many scientific applications [75] [86]-[93]. Shotgun sequencing is a widely used technique for the detection of pathogens [94]. Successful metagenomic profiling critically relies on a good DNA reference library for the organisms being identified. PathoDNA is the first test of its kind with a custom reference library to enable bio- 
informatics responsible for its remarkably high levels of accuracy and selectivity. Data from over 1000 Lyme disease patients who came to Envita during the past decade was collected to form the base of this incredible, novel reference library that allows PathoDNA to function. The detection of Borrelia and other spirochetes is an application for NGS which ought to receive more widespread attention not simply because it is a superior technology, but because the existing technologies of ELISA and Western blot are inadequate to the current needs of hundreds of thousands of people infected each year in the US alone.

The result of that research is an incredible difference in accuracy between ELISA-Western blot and PathoDNA (Next-Generation DNA Sequencing): 29\% $40 \%$ for ELISA-Western blot and $>95 \%$ for PathoDNA. In the case of B. burgdorferi specifically, PathoDNA was shown to be $98.8 \%$ accurate! PathoDNA meets and exceeds the CDC recommended accuracy threshold of $95 \%$ or greater for a diagnostic test, while the two-tier process mandated by the CDC itself does not. This is an ironic result, but the CDC is not wrong to suggest that a high level of accuracy should be expected for medical diagnostic tests since the wellbeing of hundreds of thousands of human lives often depends on sound and accurate test results. Below, Figure 6 offers a concise comparison of features between ELISA/Western blot and PathoDNA.

PathoDNA is also a broader test than ELISA-Western blot, which only looks for antibodies for a narrow range of organisms, namely specific species of Borrelia. PathoDNA utilizes a reference library of DNA containing information for 11 different species of pathogen that cause Lyme disease or are common coinfections that coincide with Chronic Lyme disease Syndrome. PathoDNA achieves many times greater accuracy and a broader scan for other types of Lyme disease

\section{Two-Tier ELISA/Western Blot vs PathoDNA}

PathoDNA's Next-Generation DNA Sequencing (NGS) utilizes bioinformatics for more accurate and efficient ways to identify the etiology of difficult to diagnose patients with unknown or complex infectious diseases.

\section{ELISA/WESTERN BLOT}

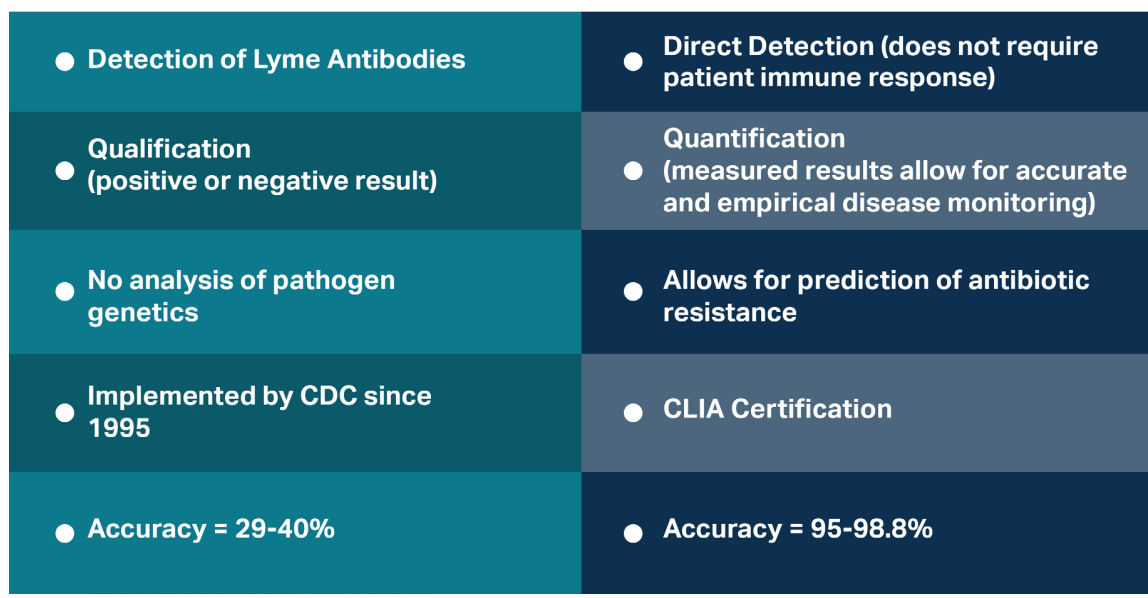

Figure 6. A comparison of key features of the two-tier test against PathoDNA [38] [61] [79]. 
and coinfections in a single step. Beyond these traits, Next-Generation DNA Sequencing allows for quantifying the infection in the form of the amount of DNA reads present in a sample. This allows a physician to track an infection's progress over time and see if it responds to treatment in an easily measurable fashion. All these qualities make PathoDNA a vastly more useful test for Lyme disease and its coinfections than the CDC recommended two-tier test.

In conclusion, the state of Lyme disease detection and diagnosis recommended by the CDC has existed for far too long and is now actively harming the population in the United States and abroad by allowing for increasing rates of Lyme to go undetected and untreated. The two-tier process of ELISA and Western blot is not sensitive or accurate enough even by the CDC's criteria and ought to be replaced by a new, high-tech detection platform. With over 95\% accuracy, the Next-generation DNA sequencing platform "PathoDNA" utilizing the Ion Torrent S5 instrument may be the solution we need to counter the growing rates of untreated and undetected Lyme disease in this country and elsewhere.

\section{Conflicts of Interest}

The authors declare no conflicts of interest regarding the publication of this paper.

\section{References}

[1] Ewig, S., et al. (2002) Factors Associated with Unknown Aetiology in Patients with Community-Acquired Pneumonia. European Respiratory Journal, 20, 1254-1262. https://doi.org/10.1183/09031936.02.01942001

[2] Bleeker-Rovers, C., et al. (2007) A Prospective Multicenter Study on Fever of Unknown Origin: The Yield of a Structured Diagnostic Protocol. Medicine, 86, 26-38. https://doi.org/10.1097/MD.0b013e31802fe858

[3] Smith, A.J., Oertle, J. and Prato, D. (2014) Environmental Carcinogens and the Kinds of Cancers They Cause. Open Journal of Oncology, 3, 1-16.

[4] Smith, A.J., Oertle, J. and Prato, D. (2014) Cancer and Infectious Causes. Open Journal of Medical Microbiology, 4, 161-177. https://doi.org/10.4236/ojmm.2014.43019

[5] Blasi, F. (2018) Lung Diseases: Chronic Respiratory Infections. International Journal of Molecular Sciences, 19, Article No. 3051.

[6] Du, Y., et al. (2019) Association between Hepatitis B Virus Infection and Chronic Kidney Disease: A Cross-Sectional Study from 3 Million Population Aged 20 to 49 Years in Rural China. Medicine, 98, e14262. https://doi.org/10.1097/MD.0000000000014262

[7] Ewald, W. (2000) Plague Time: How Stealth Infections Cause Cancers, Heart Disease, and Other Deadly Ailments. Free Press, New York.

[8] Israeli, E., et al. (2012) Guillain-Barré Syndrome-A Classical Autoimmune Disease Triggered by Infection or Vaccination. Clinical Reviews in Allergy \& Immunology, 42, 121-130. https://doi.org/10.1007/s12016-010-8213-3

[9] Rasa, S., Nora-Krukle, Z., et al. (2018) Chronic Viral Infections in Myalgic Encephalomyelitis/Chronic Fatigue Syndrome (ME/CFS). Journal of Translational Medicine, 16, Article No. 268. https://doi.org/10.1186/s12967-018-1644-y 
[10] WHO (2018) Antibiotic Resistance.

https://www.who.int/news-room/fact-sheets/detail/antibiotic-resistance

[11] Schwartz, A.M., et al. (2017) Surveillance for Lyme Disease-United States, 2008-2015. MMWR Surveillance Summaries, 66, 1-12. https://doi.org/10.15585/mmwr.ss6622a1

[12] Hofhuis, A., et al. (2015) Physician Reported Incidence of Early and Late Lyme Borreliosis. Parasites \& Vectors, 8, Article No. 161. https://doi.org/10.1186/s13071-015-0777-6

[13] Hofhuis, A., et al. (2015) Continuing Increase of Tick Bites and Lyme Disease between 1994 and 2009. Ticks and Tick-Borne Diseases, 6, 69-74. https://doi.org/10.1016/j.ttbdis.2014.09.006

[14] Miller, J.M. (2019) 2018 in Review-A Fireside Chat with a Clinical Microbiology Perspective. Clinical Microbiology Newsletter, 41, 75-81. https://doi.org/10.1016/j.clinmicnews.2019.04.003

[15] Deresinski, S. (2019) The Longhorned Tick: Is It Coming to a Place Near You? Infectious Disease Alert.

[16] Hinckley, A.F., et al. (2014) Lyme Disease Testing by Large Commercial Laboratories in the United States. Clinical Infectious Diseases, 59, 676-681. https://doi.org/10.1093/cid/ciu397

[17] Lin, S., et al. (2019) The Effects of Multiyear and Seasonal Weather Factors on Incidence of Lyme Disease and Its Vector in New York State. Science of the Total Environment, 665, 1182-1188. https://doi.org/10.1016/j.scitotenv.2019.02.123

[18] Rizzoli, A., et al. (2011) Lyme Borreliosis in Europe. Eurosurveillance, 16, pii=19906. https://doi.org/10.2807/ese.16.27.19906-en

[19] Sykes, R.A. and Makiello, P. (2016) An Estimate of Lyme Borreliosis Incidence in Western Europe. Journal of Public Health, 39, 74-81. https://doi.org/10.1093/pubmed/fdw017

[20] Hook, S., Nelson, C. and Mead, P. (2013) Self-Reported Lyme Disease Diagnosis, Treatment, and Recovery: Results from 2009, 2011, \& 2012 HealthStyles Nationwide Surveys. 13 th International Conference on Lyme Borreliosis and Other Tick-Borne Diseases, Boston, MA, 19 August 2013.

[21] Kobayashi, T., et al. (2019) Misdiagnosis of Lyme Disease with Unnecessary Antimicrobial Treatment Characterizes Patients Referred to an Academic Infectious Diseases Clinic. Open Forum Infectious Diseases, 6, ofz299. https://doi.org/10.1093/ofid/ofz299

[22] Aucott, J., et al. (2009) Diagnostic Challenges of Early Lyme Disease: Lessons from a Community Case Series. BMC Infectious Diseases, 9, Article No. 79. https://doi.org/10.1186/1471-2334-9-79

[23] Adrion, E.R., et al. (2015) Health Care Costs, Utilization and Patterns of Care Following Lyme Disease. PLOS ONE, 10, e0116767. https://doi.org/10.1371/journal.pone.0116767

[24] Zhang, X., et al. (2006) Economic Impact of Lyme Disease. Emerging Infectious Diseases, 12, 653-660. https://doi.org/10.3201/eid1204.050602

[25] Johnson, L. (2013) Insurance and Lyme Disease: A Problem of Displaced Costs. https://www.lymedisease.org/wp-content/uploads/2015/02/Chart-book-11.14.13-FI NAL-with-correction.pdf

[26] van Den Wijngaard, C.C., et al. (2017) The Cost of Lyme Borreliosis. European Journal of Public Health, 27, 538-547. https://doi.org/10.1093/eurpub/ckw269

[27] van Den Wijngaard, C.C., et al. (2015) The Burden of Lyme Borreliosis Expressed in 
Disability-Adjusted Life Years. European Journal of Public Health, 25, 1071-1078. https://doi.org/10.1093/eurpub/ckv091

[28] Kraiczy, P., et al. (2003) Immune Evasion of Borrelia burgdorferi : Mapping of a Complement-Inhibitor Factor H-Binding Site of BbCRASP-3, a Novel Member of the Erp Protein Family. European Journal of Immunology, 33, 697-707. https://doi.org/10.1002/eji.200323571

[29] Xu, Q., McShan, K. and Liang, F.T. (2007) Identification of an $\operatorname{sp} p$ C Operator Critical for Immune Evasion of Borrelia burgdorferi. Molecular Microbiology, 64, 220-231. https://doi.org/10.1111/j.1365-2958.2007.05636.x

[30] Hoxeng, K. (1997) Lyme Disease Mimics Many Other Diseases. Journal of Emergency Nursing, 23, 516. https://doi.org/10.1016/S0099-1767(97)90246-4

[31] Assadi, M. and Assadi, C.A. (1996) Lyme Disease May Mimic Lymphoma. Journal of Investigative Medicine, 44, 334.

[32] Waton, J., et al. (2007) Lyme Disease Could Mimic Dermatomyositis. La Revue de Médecine Interne, 28, 343-345. https://doi.org/10.1016/j.revmed.2007.01.002

[33] Meyerhoff, J., et al. (2019) Lyme Disease Workup: Approach Considerations, Serologic Testing, Polymerase Chain Reaction Testing. https://emedicine.medscape.com/article/330178-worku

[34] Centers for Disease Control and Prevention (2018) Two-Step Laboratory Testing Process. https://www.cdc.gov/lyme/diagnosistesting/labtest/twostep/

[35] Wharton, M., Chorba, T.L., Vogt, R.L., et al. (1990) Case Definitions for Public Health Surveillance. Morbidity and Mortality Weekly Report, 39, 19-21.

[36] Dressler, F., et al. (1993) Western Blotting in the Serodiagnosis of Lyme Disease. The Journal of Infectious Diseases, 167, 392-400.

https://doi.org/10.1093/infdis/167.2.392

[37] Centers for Disease Control and Prevention (1995) Recommendations for Test Performance and Interpretation from the Second National Conference on Serologic Diagnosis of Lyme Disease. Morbidity and Mortality Weekly Report, 44, 590-591.

[38] Sigal, L.H. (1995) Diagnosis of Lyme Disease. JAMA, 274, 1427. https://doi.org/10.1001/jama.1995.03530180021020

[39] Research Portfolio Online Reporting Tools (2021) Estimates of Funding for Various Research, Condition, and Disease Categories. https://report.nih.gov/funding/categorical-spending\#/

[40] Cook, M.J. and Puri, B.K. (2017) Application of Bayesian Decision-Making to Laboratory Testing for Lyme Disease and Comparison with Testing for HIV. International Journal of General Medicine, 10, 113-123. https://doi.org/10.2147/IJGM.S131909

[41] Coulter, P., et al. (2005) Two-Year Evaluation of Borrelia burgdorferi Culture and Supplemental Tests for Definitive Diagnosis of Lyme Disease. Journal of Clinical Microbiology, 43, 5080-5084.

[42] Wojciechowska-Koszko, I., et al. (2011) Serodiagnosis of Borreliosis: Indirect Immunofluorescence Assay, Enzyme-Linked Immunosorbent Assay and Immunoblotting. Archivum Immunologiae et Therapiae Experimentalis, 59, 69-77. https://doi.org/10.1007/s00005-010-0111-0

[43] Schutzer, S.E., et al. (1990) Sequestration of Antibody to Borrelia burgdorferi in Immune Complexes in Seronegative Lyme Disease. The Lancet, 335, 312-315. https://doi.org/10.1016/0140-6736(90)90606-6

[44] Horowitz, R.I. (2016) Approach to Diagnosing Lyme Disease Misses a Large Pro- 
portion of Cases. British Medical Journal, 352, i113.

https://doi.org/10.1136/bmj.i113

[45] Borgermans, L., et al. (2015) Lyme Disease: Time for a New Approach? British Medical Journal, 351, h6250. https://doi.org/10.1136/bmj.h6520

[46] Ang, C.W., et al. (2011) Large Differences between Test Strategies for the Detection of Anti-Borrelia Antibodies are Revealed by Comparing Eight ELISAs and Five Immunoblots. European Journal of Clinical Microbiology \& Infectious Diseases, 30, 1027-1032. https://doi.org/10.1007/s10096-011-1157-6

[47] Marangoni, A., et al. (2005) Comparative Evaluation of Three different ELISA Methods for the Diagnosis of Early Culture-Confirmed Lyme Disease in Italy. Journal of Medical Microbiology, 54, 361-367. https://doi.org/10.1099/jmm.0.45853-0

[48] Towbin, H., Staehelin, T. and Gordon, J. (1979) Electrophoretic Transfer of Proteins from Polyacrylamide Gels to Nitrocellulose Sheets: Procedure and Some Applications. Proceedings of the National Academy of Sciences of the United States of America, 76, 4350-4354. https://doi.org/10.1073/pnas.76.9.4350

[49] Liang, F.T., et al. (2000) Antigenic Conservation of an Immunodominant Invariable Region of the VlsE Lipoprotein among European Pathogenic Genospecies of Borrelia burgdorferi SL. The Journal of Infectious Diseases, 182, 1455-1462. https://doi.org/10.1086/315862

[50] Branda, J.A., et al. (2010) 2-Tiered Antibody Testing for Early and Late Lyme Disease Using Only an Immunoglobulin G Blot with the Addition of a VlsE Band as the Second-Tier Test. Clinical Infectious Diseases, 50, 20-26. https://doi.org/10.1086/648674

[51] Susan, E., Sharp, B.B., Gaynor, A., Owen, M., Parker, M., Pentella, M., Randall, L., Robeson, B., Werner, B., Wesolowski, L. and Wroblewski, K. (2015) Limitations for the Use of HIV-1 Western Blot in Plasma/Serum. American Society for Microbiology.

[52] Parekh, B.S., et al. (2019) Diagnosis of Human Immunodeficiency Virus Infection. Clinical Microbiology Reviews, 32, e00064.

[53] Horowitz, R. and Freeman, R. (2018) Improvement of Common Variable Immunodeficiency Using Embryonic Stem Cell Therapy in a Patient with Lyme Disease: A Clinical Case Report. Clinical Case Reports, 6, 1166-1171. https://doi.org/10.1002/ccr3.1556

[54] Wang, G.Q., et al. (1999) Molecular Typing of Borrelia burgdorferiSensu Lato: Taxonomic, Epidemiological, and Clinical Implications. Clinical Microbiology Reviews, 12, 633-653.

[55] Wilske, B. (2003) Diagnosis of Lyme Borreliosis in Europe. Vector-Borne and Zoonotic Diseases, 3, 215-227. https://doi.org/10.1089/153036603322662200

[56] Schoen, R.T. (2013) Editorial Commentary: Better Laboratory Testing for Lyme Disease: No More Western Blot. Clinical Infectious Diseases, 57, 341-343. https://doi.org/10.1093/cid/cit238

[57] Robertson, J., et al. (2000) A European Multicenter Study of Immunoblotting in Serodiagnosis of Lyme Borreliosis. Journal of Clinical Microbiology, 38, 2097-2102.

[58] Norman, G.L., et al. (1996) Serodiagnosis of Lyme Borreliosis by Borrelia burgdorferi sensu stricto, B. garinii, and B. afzelii Western Blots (Immunoblots). Journal of Clinical Microbiology, 34, 1732-1738.

[59] Igenex. Results that Matter: Interpreting Test Results. https://igenex.com/test-interpretations/ 
[60] Hurley, D. and Santora, M. (2005) Unproved Lyme Disease Tests Prompt Warnings. New York Times.

[61] Aguero-Rosenfeld, M.E., et al. (2005) Diagnosis of Lyme Borreliosis. Clinical Microbiology Reviews, 18, 484-509.

[62] Engvall, E. and Perlmann, P. (1972) Enzyme-Linked Immunosorbent Assay, ELISA. In: Peeters, H., Ed., Protides of the Biological Fluids, Pergamon, Oxford, 553-556. https://doi.org/10.1016/B978-0-08-016876-0.50102-X

[63] Kragstrup, T., et al. (2013) A Simple Set of Validation Steps Identifies and Removes False Results in a Sandwich Enzyme-Linked Immunosorbent Assay Caused by Anti-Animal IgG Antibodies in Plasma from Arthritis Patients. SpringerPlus, 2, Article No. 263. https://doi.org/10.1186/2193-1801-2-263

[64] Wormser, G., et al. (2013) Single-Tier Testing with the C6 Peptide ELISA Kit Compared with Two-Tier Testing for Lyme Disease. Diagnostic Microbiology \& Infectious Disease, 75, 9-15. https://doi.org/10.1016/j.diagmicrobio.2012.09.003

[65] Busson, L., et al. (2012) Evaluation of Commercial Screening Tests and Blot Assays for the Diagnosis of Lyme Borreliosis. Diagnostic Microbiology \& Infectious Disease, 73, 246-251. https://doi.org/10.1016/j.diagmicrobio.2012.04.001

[66] Newton, C.R. and Graham, A. (1997) PCR (Introduction to Biotechniques Series). 2nd Edition, BIOS Scientific Publishers, Oxford.

[67] Schochetman, G., Ou, C.Y. and Jones, W.K. (1988) Polymerase Chain Reaction. The Journal of Infectious Diseases, 158, 1154-1157.

https://doi.org/10.1093/infdis/158.6.1154

[68] Skogman, B.H., et al. (2021) Lyme Neuroborreliosis in Swedish Children-PCR as a Complementary Diagnostic Method for Detection of Borrelia burgdorferi sensu lato in Cerebrospinal Fluid. European Journal of Clinical Microbiology \& Infectious Diseases, 40, 1003-1012. https://doi.org/10.1007/s10096-020-04129-7

[69] Dessau, R.B., et al. (2018) To Test or Not to Test? Laboratory Support for the Diagnosis of Lyme Borreliosis: A Position Paper of ESGBOR, the ESCMID Study Group for Lyme Borreliosis. Clinical Microbiology and Infection, 24, 118-124.

https://doi.org/10.1016/j.cmi.2017.08.025

[70] Nocton, J.J., et al. (1996) Detection of Borrelia burgdorferi DNA by Polymerase Chain Reaction in Cerebrospinal Fluid in Lyme Neuroborreliosis. The Journal of Infectious Diseases, 174, 623-627. https://doi.org/10.1093/infdis/174.3.623

[71] Nocton, J.J., et al. (1994) Detection of Borrelia burgdorferi DNA by Polymerase Chain Reaction in Synovial Fluid from Patients with Lyme Arthritis. The New England Journal of Medicine, 330, 229-234. https://doi.org/10.1056/NEJM199401273300401

[72] Chiu, C.Y. (2013) Viral Pathogen Discovery. Current Opinion in Microbiology, 16, 468-478. https://doi.org/10.1016/j.mib.2013.05.001

[73] Cummings, L.A., et al. (2016) Clinical Next Generation Sequencing Outperforms Standard Microbiological Culture for Characterizing Polymicrobial Samples. Clinical Chemistry, 62, 1465-1473. https://doi.org/10.1373/clinchem.2016.258806

[74] Salipante, S.J., et al. (2014) Coinfection of Fusobacterium nucleatum and Actinomyces israelii in Mastoiditis Diagnosed by Next-Generation DNA Sequencing. Journal of Clinical Microbiology, 52, 1789-1792.

[75] Deurenberg, R.H., et al. (2017) Application of Next Generation Sequencing in Clinical Microbiology and Infection Prevention. Journal of Biotechnology, 243, 16-24. https://doi.org/10.1016/j.jbiotec.2016.12.022 
[76] Salipante, S.J., et al. (2015) Application of Whole-Genome Sequencing for Bacterial Strain Typing in Molecular Epidemiology. Journal of Clinical Microbiology, 53, 1072-1079.

[77] Sahoo, M.K., et al. (2013) Detection of Cytomegalovirus Drug Resistance Mutations by Next-Generation Sequencing. Journal of Clinical Microbiology, 51, 3700-3710.

[78] Popp, J. and Bauer, M. (2015) Modern Techniques for Pathogen Detection. Wiley Blackwell, Weinheim. https://doi.org/10.1002/9783527687978

[79] Meyers, G. (2021) Validation of Ion Torrent Tick-Borne Pathogen Panel. Next Bio-Research Services, LLC., Virginia.

[80] Tatusov, R., et al. (2003) The COG Database: An Updated Version Includes Eukaryotes. BMC Bioinformatics, 4, Article No. 41.

[81] Kanehisa, M., et al. (2004) The KEGG Resource for Deciphering the Genome. Nucleic Acids Research, 32, D277-D280. https://doi.org/10.1093/nar/gkh063

[82] FDA (2018) Laboratory Developed Tests. https://www.fda.gov/medical-devices/vitro-diagnostics/laboratory-developed-tests

[83] FDA (2017) Commercial Laboratory Tests and FDA Approval. https://labtestsonline.org/articles/commercial-tests-fda-approval

[84] Thacker, S.B., Sencer, D.J. and Jaffe, H.W. (2017) Centers for Disease Control. In: Quah, S.R., Ed., International Encyclopedia of Public Health, Academic Press, Cambridge, 448-454. https://doi.org/10.1016/B978-0-12-803678-5.00057-6

[85] Tuller, D. (2019) Trial by Error: The CDC’s Pathetic Response to Reuters. https://www.virology.ws/2019/03/25/trial-by-error-the-cdcs-pathetic-response-to-re uters/

[86] Sato, M., et al. (2016) Acute Encephalopathy in an Immunocompromised Boy with Astrovirus-MLB1 Infection Detected by Next Generation Sequencing. Journal of Clinical Virology, 78, 66-70. https://doi.org/10.1016/j.jcv.2016.03.010

[87] Tarabichi, M., et al. (2018) Diagnosis of Streptococcus canis Periprosthetic Joint Infection: The Utility of Next-Generation Sequencing. Arthroplasty Today, 4, 20-23. https://doi.org/10.1016/j.artd.2017.08.005

[88] Tarabichi, M., et al. (2018) Diagnosis of Periprosthetic Joint Infection: The Potential of Next-Generation Sequencing. The Journal of Bone and Joint Surgery, 100, 147-154. https://doi.org/10.2106/JBJS.17.00434

[89] Be, N.A., et al. (2014) Microbial Profiling of Combat Wound Infection through Detection Microarray and Next-Generation Sequencing. Journal of Clinical Microbiology, 52, 2583-2594.

[90] Mouraviev, V. and McDonald, M.W. (2018) MP23-06 an Utilization of Next Generation Sequencing of Urine Samples for Monitoring of Urinary Tract Infection in Patients with Neurogenic Bladder. The Journal of Urology, 199, e283. https://doi.org/10.1016/j.juro.2018.02.738

[91] Ogishi, M., et al. (2014) Next-Generation Sequencing Revealed the Prevalence of Multi-Geno/Subtypic Multiple Infection of Hepatitis C Virus in Hemophiliac Patients in Japan. Hepatology, 60, 897A-897A.

[92] Kothari, A., et al. (2017) The Role of Next Generation Sequencing in Infection Prevention in Human Parainfluenza Virus 3 Infections in Immunocompromised $\mathrm{Pa}$ tients. Journal of Clinical Virology, 92, 53-55.

https://doi.org/10.1016/j.jcv.2017.05.010

[93] Wollants, E., et al. (2018) Use of Next-Generation Sequencing for Diagnosis of West Nile Virus Infection in Patient Returning to Belgium from Hungary. Emerging In- 
fectious Diseases, 24, 2380-2382. https://doi.org/10.3201/eid2412.180494

[94] Yang, X., et al. (2016) Use of Metagenomic Shotgun Sequencing Technology to Detect Foodborne Pathogens within the Microbiome of the Beef Production Chain. Applied and Environmental Microbiology, 82, 2433-2443. 